\title{
Clinical and Histologic Features of Patients with Biopsy-Proven Metabolic Dysfunction-Associated Fatty Liver Disease
}

\author{
Shang-Chin Huang ${ }^{1}$, Hau-Jyun Su${ }^{1}$, Jia-Horng Kao ${ }^{1,2,3}$, Tai-Chung Tseng ${ }^{1,3}$, Hung-Chih Yang ${ }^{1,3}$, Tung-Hung Su ${ }^{1,3}$, \\ Pei-Jer Chen ${ }^{1,2,3}$, and Chun-Jen Liu' ${ }^{1,2,3}$ \\ ${ }^{1}$ Department of Internal Medicine, ${ }^{2}$ Graduate Institute of Clinical Medicine, and ${ }^{3}$ Hepatitis Research Center, National Taiwan University \\ College of Medicine and Hospital, Taipei City, Taiwan
}

\section{Article Info \\ Received July 14, 2020 \\ Revised September 16, 2020 \\ Accepted September 24, 2020 \\ Published online January 13, 2021 \\ Corresponding Author \\ Chun-Jen Liu \\ ORCID https://orcid.org/0000-0002-6202-0993 \\ E-mail cjliu@ntu.edu.tw}

Shang-Chin Huang and Hau-Jyun Su contributed equally to this work as first authors.
Background/Aims: Fatty liver disease is defined as a cluster of diseases with heterogeneous etiologies, and its definition continues to evolve. The novel conceptional criteria for metabolic dysfunction-associated fatty liver disease (MAFLD) were proposed in 2020 to avoid the exclusion of a certain subpopulation, but their evaluations have been limited. We aimed to examine and compare the clinical as well as histologic features of MAFLD versus nonalcoholic fatty liver disease (NAFLD) in patients with biopsy-proven hepatic steatosis.

Methods: From January 2009 to December 2019, 175 patients with histology-proven hepatic steatosis and 10 with cryptogenic cirrhosis who were treated at National Taiwan University Hospital, Taipei, Taiwan, were enrolled. Patients were classified into different groups according to the diagnostic criteria of MAFLD and NAFLD. The clinical and histologic features were then analyzed and compared.

Results: In total, 76 patients (41.1\%) were diagnosed with both MAFLD and NAFLD, 81 patients $(43.8 \%)$ were diagnosed with MAFLD alone, nine patients $(4.9 \%)$ were diagnosed with NAFLD alone, and 19 patients $(10.3 \%)$ were diagnosed with neither. Those with MAFLD alone exhibited a higher degree of disease severity regarding histology and laboratory data than those with NAFLD alone. Advanced fibrosis was associated with the presences of hepatitis $B$ virus infection and metabolic diseases.

Conclusions: The novel diagnostic criteria for MAFLD include an additional $38.9 \%$ of patients with hepatic steatosis and can better help identify those with a high degree of disease severity for early intervention than can the previous NAFLD criteria. (Gut Liver 2021;15:451-458)

Key Words: Metabolic dysfunction-associated fatty liver disease; Nonalcoholic fatty liver disease; Metabolic diseases; Hepatic fibrosis; Hepatitis B

\section{INTRODUCTION}

Hepatic steatosis has been one of the major etiologies for chronic liver diseases with steadily increasing prevalence especially for those in the urbanized countries. ${ }^{1,2} \mathrm{Un}$ fortunately, in the past years, the international guidelines defined the "nonalcoholic fatty liver disease (NAFLD)" and "nonalcoholic steatohepatitis (NASH)" by excluding other causes of hepatic steatosis, such as alcoholism, pregnancy, hepatitis $\mathrm{C}$ virus (HCV) infection and others. ${ }^{3-5}$ Because several metabolic and chronic diseases are known to be associated with the development of fatty liver disease, the influence and interactions of these chronic or metabolic diseases with hepatic steatosis would not be able to clarify using traditional diagnostic criteria. The expanding criteria of inclusion rather than exclusion are expected to make a more comprehensive overview of the widespread hepatic steatosis disease. A new positive definition for the metabolic dysfunction-associated fatty liver disease (MAFLD) was thus proposed from an international expert consensus in 2020. The diagnosis of MAFLD is made if the patient has hepatic steatosis along with one of the three criteria, name- 
ly overweight/obesity, presence of type 2 diabetes mellitus (DM), and at least two metabolic risk abnormalities. ${ }^{6}$

Since the new definition for MAFLD is a conceptional one, there are debates on the change in terminology, and only very limited information about its application in the real world. ${ }^{8}$ A retrospective study with population-based data from the National Health and Nutrition Examination Surveys in United State during 1988 to 1994 is the only one so far providing the test and validation of the new criteria in clinical practice. ${ }^{8}$ However, the histologic features were not available in the study. Although a liver biopsy is not required for the diagnosis of MAFLD, provision of liver histology will accurately assess the degree of steatosis as well as the severity of fibrosis, and will be of great help to evaluate the major difference between the prior NAFLD and the novel MAFLD criteria, and the potential clinical impact of new MAFLD diagnostic criteria. Besides, the clinical applications of this new definition in hepatitis B virus (HBV) endemic countries also await further studies to clarify. In this study, we thus aimed to investigate the clinical as well as the histologic features of MAFLD, and compared the NAFLD and MAFLD criteria in patients with biopsyproven hepatic steatosis.

\section{MATERIALS AND METHODS}

\section{Study population}

From January 2009 to December 2019, a total of 780 percutaneous ultrasound-guided liver random biopsies at the National Taiwan University Hospital, Taipei, Taiwan was retrospectively collected. Of these patients, 175 patients with hepatic steatosis and 10 with cryptogenic cirrhosis in the histological reports were identified for evaluation (Fig. 1). This study was approved by the Institutional Review Board of National Taiwan University Hospital (IRB number: NTUH 201909063RINA) and conformed to the ethical guidelines of the 1975 Declaration of Helsinki. The informed consent was waived.

\section{Data collection}

A standardized record form was used for data collection. Retrospective review of the medical records, reports, and images was performed. Age, gender, comorbidity, alcohol consumption, and medication history were recorded. The body mass index (BMI) was calculated as weight in kilograms divided by height in meters squared. Laboratory data, such as platelet count, prothrombin time, international normalized ratio, alanine aminotransferas, aspartate aminotransferase, alkaline phosphatase, gamma glutamyl transferase, total bilirubin, fasting glucose, hemoglobin A1c, total cholesterol, triglyceride, autoimmune antibodies and the seromarkers for viral hepatitis were collected. The fibrosis-4 score and aspartate aminotransferase to platelet ratio index were also analyzed.

\section{Histology assessment}

The histologic reports of the patients were recorded, including the grading of steatosis, lobular inflammation and ballooning change according to the NASH Clinical Research

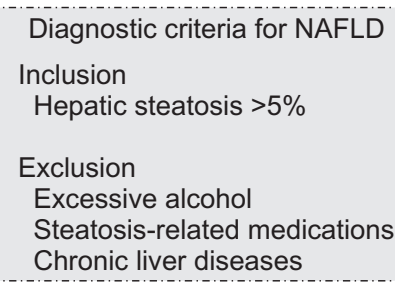

A total of 780 percutaneous ultrasoundguided liver random biopsies at the National Taiwan University Hospital during 2009-2019

175 Patients with hepatic steatosis 10 Patients with cryptogenic cirrhosis
Diagnostic criteria for MAFLD

Hepatic steatosis $>5 \%$ $+$

One of the following three: Overweight/obesity (BMI $\geq 23 \mathrm{~kg} / \mathrm{m}^{2}$ ) Type 2 diabetes mellitus $\geq 2$ metabolic risk abnormalities

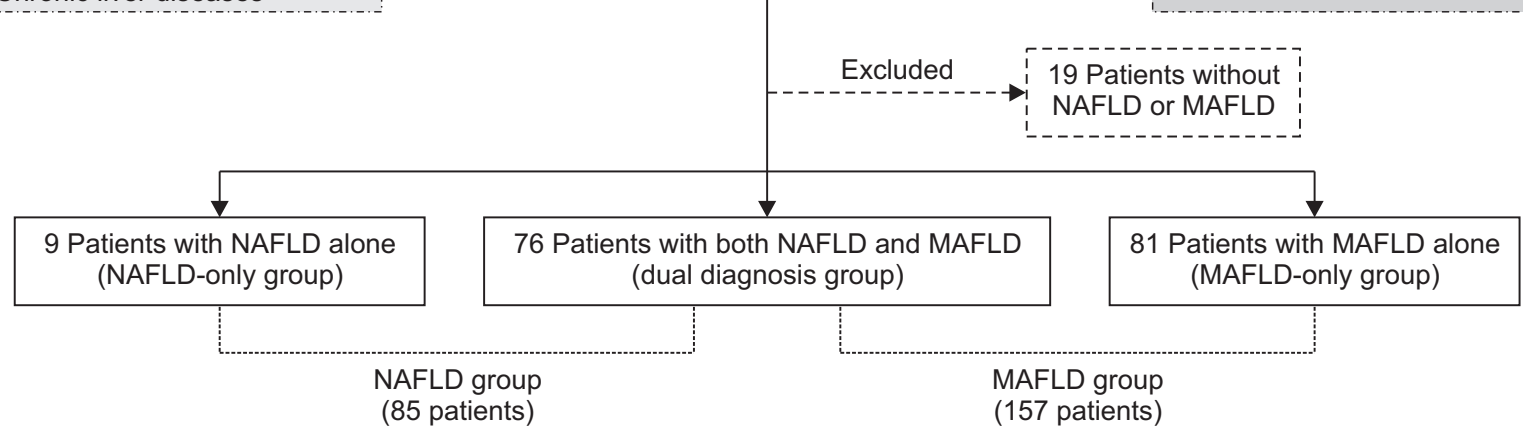

Fig. 1. Flowchart of patient selection and the diagnostic criteria for metabolic dysfunction-associated fatty liver disease (MAFLD) and nonalcoholic fatty liver disease (NAFLD).

$\mathrm{BMI}$, body mass index. 
Network (NASH CRN). The NAFLD Activity Score (NAS) was calculated. ${ }^{9}$ The degree of hepatic fibrosis was stratified according to the NASH CRN fibrosis staging system. Hepatic fibrosis with stage $0-2$ was defined as early fibrosis while that with stage $3-4$ was defined as advanced fibrosis. Specimens with the diagnosis of cryptogenic cirrhosis were reviewed by a consulted liver pathologist additionally.

\section{Diagnostic criteria for MAFLD}

The diagnosis of MAFLD was made if there were evidence of hepatic steatosis plus one of the following three criteria, namely overweight/obesity (BMI $\geq 23 \mathrm{~kg} / \mathrm{m}^{2}$ in Asians), type $2 \mathrm{DM}$, or evidence of metabolic dysregulation $(\geq 2$ metabolic risk abnormalities as follows: waist circumference $\geq 90 / 80 \mathrm{~cm}$ in Asian men and women, blood pressure $\geq 130 / 85 \mathrm{~mm} \mathrm{Hg}$ or specific drug treatment, plasma triglycerides $\geq 150 \mathrm{mg} / \mathrm{dL}$ or specific drug treatment, plasma high-density lipoprotein-cholesterol $<40 \mathrm{mg} / \mathrm{dL}$ for men and $<50 \mathrm{mg} / \mathrm{dL}$ for women or specific drug treatment, prediabetes, homeostasis model assessment of insulin resistance score $\geq 2.5$, and plasma high-sensitivity Creactive protein level $>2 \mathrm{mg} / \mathrm{L}$ ). ${ }^{6}$ The diagnostic criteria for MAFLD-related cirrhosis in those without typical histological steatosis were past or present evidence of metabolic risk factors compatible with the criteria for MAFLD, with documentation of MAFLD on a previous liver biopsy or historical documentation of steatosis by hepatic imaging. ${ }^{6}$

\section{Diagnostic criteria for NAFLD}

The diagnosis of NAFLD was made if there was presence of hepatic steatosis $>5 \%$ of all hepatocytes on the histologic report without the history of excessive alcohol intake (alcohol consumption $>140 \mathrm{~g}$ per week for men and $>70$ g per week for women), the usage of steatosis-related medications or chronic liver diseases. The diagnosis of NAFLD-related cirrhosis was made if there was pathological presentation of NAFLD, or with comorbidity of BMI $>30 \mathrm{~kg} / \mathrm{m}^{2}$, hypertension or type $2 \mathrm{DM}$, and unexplained by other etiology of chronic liver disease.

\section{Comparison of the MAFLD and NAFLD criteria}

In order to demonstrate the difference between the MAFLD and NAFLD criteria, we defined patients meeting the MAFLD but not NAFLD criteria as the "MAFLD-only group" those meeting the NAFLD rather than MAFLD criteria as the "NAFLD-only group," and those meeting both MAFLD/ NAFLD criteria (Fig. 1). The comparison of the clinical and histologic data was conducted among the three groups and between the MAFLD-only group and NAFLD-only group.

\section{Statistical analysis}

The categorical data was compared by the chi-square and two-tailed Fisher exact tests. The continuous variables were examined by the two-sample t-test. A two-tailed $\mathrm{p}<0.05$ was considered statistically significant. The presences of comorbidities, namely hypertension, type $2 \mathrm{DM}$, dyslipidemia, HBV infection, HCV infection and alcoholism, were comprehensively included in the logistic regression analyses to determine the association with advanced fibrosis in patients with MAFLD. Comorbidities with $\mathrm{p}<0.1$ in the univariate analyses were used in a multivariate logistic regression model. The statistical analyses were conducted by PASW Statistics for Windows, version 18.0 (SPSS Inc., Chicago, IL, USA).

\section{RESULTS}

\section{Patient demographics, characteristics and laboratory data}

There were 81 (43.8\%) patients fitting the MAFLD criteria alone, nine (4.9\%) fitting the NAFLD criteria alone, and $76(41.1 \%)$ fitting the both criteria while $19(10.3 \%)$ not fitting either one (Fig. 1). The indications for liver biopsies in each group are listed in Table 1. In the MAFLDonly group, the reasons for exclusion from NAFLD are demonstrated in Table 2.

The characteristics of the patients are shown in Table 3. Among patients with dual diagnosis $(n=76)$, the percent-

Table 1. Indications for Liver Biopsies $(n=166)$

\begin{tabular}{lccc}
\hline \multicolumn{1}{c}{ Indications } & $\begin{array}{c}\text { Dual diagnosis with } \\
\text { MAFLD and NAFLD (n=76) }\end{array}$ & $\begin{array}{c}\text { MAFLD-only } \\
\text { (n=81) }\end{array}$ \\
\hline Abnormal liver functions & & & \\
Hepatocellular injury & $53(69.7)$ & $42(51.9)$ \\
Cholestatic injury & $6(7.9)$ & $8(9.9)$ & $10(12.3)$ \\
Hyperbilirubinemia & $3(3.9)$ & $3(3.7)$ & 0 \\
Unknown cirrhosis & $13(17.1)$ & $14(17.3)$ & 0 \\
Evaluation of fibrosis & 0 & $4(4.9)$ & 0 \\
Hepatic tumor & $1(1.3)$ & $1(11.1)$ &
\end{tabular}

Data are presented as number (\%).

MAFLD, metabolic dysfunction-associated fatty liver disease; NAFLD, nonalcoholic fatty liver disease. 
age of male and female gender were identical. The mean age was 51.26 years and the mean BMI was $27.65 \mathrm{~kg} / \mathrm{m}^{2}$. Some patients had comorbidities of cirrhosis (21.1\%), hypertension (48.7\%), DM (34.2\%) and dyslipidemia (40.8\%).

Table 2. Reasons for NAFLD Exclusion in Patients with MAFLD Alone ( $\mathrm{n}=81$ )

\begin{tabular}{lc}
\hline \multicolumn{1}{c}{ Reasons for exclusion } & No. (\%) \\
\hline HBV infection* & $35(43.2)$ \\
Culprit medications & $23(28.4)$ \\
HCV infection & $10(12.3)$ \\
Autoimmune liver diseases & $7(8.6)$ \\
Alcoholism & $5(6.2)$ \\
HEV infection & $1(1.2)$ \\
\hline
\end{tabular}

NAFLD, nonalcoholic fatty liver disease; MAFLD, metabolic dysfunction-associated fatty liver disease; HBV, hepatitis B virus; HCV, hepatitis $\mathrm{C}$ virus; $\mathrm{HEV}$, hepatitis $\mathrm{E}$ virus.

* Seven patients with concomitant HBV infection were excluded for having other etiologies: three with culprit medications, two with $\mathrm{HCV}$ coinfection and two with alcoholism.
None had HBV infection, HCV infection and alcoholism because of exclusion by NAFLD criteria. The mean levels of the laboratory data were also demonstrated. The mean fibrosis-4 score was 2.44 while the aspartate aminotransferase to platelet ratio index was 1.90 .

The patients meeting only one of the NAFLD or MAFLD criteria were analyzed to compare the difference between the two distinct definitions. There were $81 \mathrm{pa}$ tients in the MAFLD-only group and nine in the NAFLDonly group. There was no significant difference ( $p>0.05)$ in the gender and age, but the BMI was higher in the MAFLD-only group than that in the NAFLD-only group, 27.31 versus $21.36 \mathrm{~kg} / \mathrm{m}^{2}(\mathrm{p}<0.001)$, respectively. Among the 81 patients in the MAFLD-only group, there were 19 (23.5\%) with cirrhosis, 30 (37.0\%) with hypertension, 26 (32.1\%) with type $2 \mathrm{DM}, 16$ (19.8\%) with dyslipidemia, 42 (51.9\%) with HBV infection, 10 (12.3\%) with HCV infection, and five (6.2\%) with alcoholism. In the NAFLDonly group, there was no patient with above comorbidi-

Table 3. Demographics, Characteristics and Laboratory Data ( $n=166)$

\begin{tabular}{|c|c|c|c|c|}
\hline \multirow{2}{*}{ Variable } & \multirow{2}{*}{$\begin{array}{l}\text { Dual diagnosis with } \\
\text { MAFLD and NAFLD ( } n=76)\end{array}$} & \multicolumn{3}{|c|}{ Single diagnosis } \\
\hline & & MAFLD-only ( $n=81$ ) & NAFLD-only ( $n=9$ ) & p-value* \\
\hline \multicolumn{5}{|l|}{ Demographics } \\
\hline Male sex & $38(50.0)$ & 49 (60.5) & $4(44.4)$ & 0.479 \\
\hline Age, yr & $51.26 \pm 15.29$ & $51.93 \pm 13.44$ & $44.11 \pm 15.31$ & 0.106 \\
\hline $\mathrm{BMI}, \mathrm{kg} / \mathrm{m}^{2}$ & $27.65 \pm 4.31$ & $27.31 \pm 3.58$ & $21.36 \pm 1.59$ & $<0.001$ \\
\hline \multicolumn{5}{|l|}{ Comorbidities } \\
\hline Cirrhosis & 16 (21.1) & 19 (23.5) & 0 & 0.195 \\
\hline Hypertension & $37(48.7)$ & $30(37.0)$ & 0 & 0.027 \\
\hline Diabetes mellitus & 26 (34.2) & $26(32.1)$ & 0 & 0.055 \\
\hline Dyslipidemia & $31(40.8)$ & $16(19.8)$ & 0 & 0.353 \\
\hline HBV infection & 0 & 42 (51.9) & 0 & 0.003 \\
\hline HCV infection & 0 & 10 (12.3) & 0 & 0.590 \\
\hline Alcoholism & 0 & $5(6.2)$ & 0 & 1.000 \\
\hline \multicolumn{5}{|l|}{ Laboratory data } \\
\hline Platelet, $\times 10^{3} / \mu \mathrm{L}$ & $238.67 \pm 96.11$ & $198.64 \pm 81.48$ & $271.56 \pm 40.36$ & 0.010 \\
\hline INR & $0.99 \pm 0.07$ & $1.01 \pm 0.09$ & $0.97 \pm 0.06$ & 0.231 \\
\hline AST, U/L & $128.38 \pm 280.78$ & $98.96 \pm 91.99$ & $107.56 \pm 151.48$ & 0.805 \\
\hline$A L T, U / L$ & $152.79 \pm 182.82$ & $125.94 \pm 147.29$ & $139.56 \pm 106.52$ & 0.789 \\
\hline$A L P, U / L$ & $121.37 \pm 142.50$ & $130.18 \pm 162.64$ & $121.00 \pm 62.52$ & 0.868 \\
\hline GGT, U/L & $184.51 \pm 394.20$ & $209.03 \pm 310.67$ & $108.00 \pm 86.91$ & 0.338 \\
\hline Total bilirubin, $\mathrm{mg} / \mathrm{dL}$ & $1.59 \pm 3.12$ & $3.09 \pm 6.62$ & $0.70 \pm 0.29$ & 0.002 \\
\hline Fasting glucose, mg/dL & $124.02 \pm 50.64$ & $120.28 \pm 48.07$ & $118.33 \pm 24.50$ & 0.945 \\
\hline $\mathrm{HbA} 1 \mathrm{c}, \%$ & $6.37 \pm 1.17$ & $6.40 \pm 1.46$ & $5.00 \pm 0.00$ & 0.345 \\
\hline Total cholesterol, mg/dL & $191.73 \pm 37.98$ & $192.48 \pm 96.34$ & $208.00 \pm 63.17$ & 0.786 \\
\hline Triglyceride, mg/dL & $161.87 \pm 84.85$ & $159.24 \pm 122.18$ & $101.00 \pm 41.58$ & 0.420 \\
\hline FIB-4 score & $2.44 \pm 2.50$ & $3.02 \pm 2.99$ & $1.65 \pm 2.53$ & 0.189 \\
\hline APRI & $1.90 \pm 4.15$ & $1.89 \pm 1.83$ & $1.48 \pm 2.48$ & 0.547 \\
\hline
\end{tabular}

Data are presented as number (\%) or mean \pm SD.

MAFLD, metabolic dysfunction-associated fatty liver disease; NAFLD, nonalcoholic fatty liver disease; BMI, body mass index; HBV, hepatitis B virus; HCV, hepatitis C virus; INR, international normalized ratio; AST, aspartate aminotransferase; ALT, alanine aminotransferase; ALP, alkaline phosphatase; GGT, gamma glutamyl transferase; HbA1c, hemoglobin A1c; FIB-4, fibrosis-4; APRI, aspartate aminotransferase to platelet ratio index.

*The statistical analysis and comparison were conducted between the MAFLD-only and NAFLD-only groups. 
ties. There was no significant difference in the levels of international normalized ratio, aspartate aminotransferase, alanine aminotransferase, alkaline phosphatase, gamma glutamyl transferase, fasting glucose, hemoglobin A1c, total cholesterol, triglyceride, fibrosis- 4 score and aspartate aminotransferase to platelet ratio index between the two groups. However, the platelet count $\left(198.64 \times 10^{3} / \mu \mathrm{L}\right.$ vs $\left.271.56 \times 10^{3} / \mu \mathrm{L}, \mathrm{p}=0.010\right)$ was significantly lower and the level of total bilirubin $(3.09 \mathrm{mg} / \mathrm{dL}$ vs $0.70 \mathrm{mg} / \mathrm{dL}, \mathrm{p}=0.002)$ was significantly higher in the MAFLD-only group than those in the NAFLD-only group.

\section{Histologic features}

The histologic degree of steatosis and fibrosis is listed in Table 4. In patients with dual diagnosis, those with steatosis grade $0-1,2$, and 3 were $56.6 \%, 25.0 \%$, and $18.4 \%$. Those with lobular inflammation grade 0 to 2 were $32.9 \%$, $43.4 \%$, and $23.7 \%$. None had lobular inflammation grade 3 . The patients with ballooning change grade 0,1 , and 2-3 were $28.9 \%, 55.3 \%$, and $15.8 \%$. The mean NAS was 3.38 . There were $28(36.8 \%)$ patients with advanced fibrosis according to the NASH CRN system.

The histologic features in the MAFLD-only and NAFLD-only groups were compared. Those with steatosis grade $0-1,2$, and 3 were $65.4 \%, 23.5 \%$, and $11.1 \%$ in the MAFLD-only group, and all the nine patients in the NAFLD-only group had steatosis grade $0-1(\mathrm{p}=0.178)$. Those with lobular inflammation grade 0 to 2 were $44.4 \%$, $39.5 \%$, and $16.0 \%$ in the MAFLD-only group, and $66.7 \%$, $22.2 \%$, and $11.1 \%$ in the NAFLD-only group, respectively $(\mathrm{p}=0.516)$. None in both groups had lobular inflammation grade 3 . The patients with ballooning change grade 0,1 , and $2-3$ were $45.7 \%, 43.2 \%$, and $11.1 \%$ in the MAFLDonly group, and $77.8 \%, 22.2 \%$, and $0.0 \%$ in the NAFLDonly group, respectively $(\mathrm{p}=0.203)$. The NAS was significantly higher in the MAFLD-only group than that in the NAFLD-only group ( 2.83 vs $1.67, \mathrm{p}=0.009$ ), respectively. There were more patients with advanced fibrosis according to the NASH CRN system in the MAFLD-only group than in the NAFLD-only group, $48.1 \%$ versus $0.0 \%(\mathrm{p}=0.005)$.

\section{Comorbidities associated with advanced fibrosis in MAFLD}

In the univariate analysis of association between comorbidities and advanced fibrosis in patients with MAFLD, the presences of hypertension, DM and HBV infection were associated with more advanced fibrosis, while the presence of dyslipidemia was associated with less advanced fibrosis (Table 5). The above comorbidities were then evaluated using a multivariate logistic regression model. Hypertension (odds ratio [OR], 2.051; $\mathrm{p}=0.047$ ), $\mathrm{DM}$ (OR, 2.489; $\mathrm{p}=0.020)$ and $\mathrm{HBV}$ infection $(\mathrm{OR}, 2.447 ; \mathrm{p}=0.024)$ were independently associated with more advanced fibrosis, and dyslipidemia was independently associated with less advanced fibrosis (OR, 0.291; $\mathrm{p}=0.003$ ).

\section{DISCUSSION}

The newly-proposed definition of MAFLD focuses on the "positive" diagnostic criteria and emphasizes the role and contribution of metabolic dysfunction in fatty liver

Table 4. Histologic Degree of Steatosis and Fibrosis $(n=166)$

\begin{tabular}{|c|c|c|c|c|}
\hline \multirow{2}{*}{ Variable } & \multirow{2}{*}{$\begin{array}{l}\text { Dual diagnosis with } \\
\text { MAFLD and NAFLD ( } n=76)\end{array}$} & \multicolumn{3}{|c|}{ Single diagnosis } \\
\hline & & MAFLD-only ( $\mathrm{n}=81$ ) & NAFLD-only (n=9) & $p$-value* \\
\hline Steatosis & & & & 0.178 \\
\hline Grade $0-1$ & $43(56.6)$ & $53(65.4)$ & $9(100.0)$ & \\
\hline Grade 2 & $19(25.0)$ & $19(23.5)$ & 0 & \\
\hline Grade 3 & $14(18.4)$ & $9(11.1)$ & 0 & \\
\hline Lobular inflammation & & & & 0.516 \\
\hline Grade 0 & 25 (32.9) & $36(44.4)$ & $6(66.7)$ & \\
\hline Grade 1 & $33(43.4)$ & 32 (39.5) & $2(22.2)$ & \\
\hline Grade 2 & $18(23.7)$ & $13(16.0)$ & $1(11.1)$ & \\
\hline Grade 3 & 0 & 0 & 0 & \\
\hline Ballooning change & & & & 0.203 \\
\hline Grade 0 & $22(28.9)$ & $37(45.7)$ & $7(77.8)$ & \\
\hline Grade 1 & 42 (55.3) & 35 (43.2) & $2(22.2)$ & \\
\hline Grade 2-3 & $12(15.8)$ & $9(11.1)$ & 0 & \\
\hline NAS & $3.38 \pm 1.63$ & $2.83 \pm 1.59$ & $1.67 \pm 1.00$ & 0.009 \\
\hline Advanced fibrosis (stage 3-4) & $28(36.8)$ & $39(48.1)$ & 0 & 0.005 \\
\hline
\end{tabular}

Data are presented as number $(\%)$ or mean \pm SD.

MAFLD, metabolic dysfunction-associated fatty liver disease; NAFLD, nonalcoholic fatty liver disease; NAS, NAFLD Activity Score.

*The statistical analysis and comparison were conducted between the MAFLD-only and NAFLD-only groups. 
Table 5. Comorbidities Associated with Advanced Fibrosis (Stage 3-4) in Patients with MAFLD ( $n=157$ )

\begin{tabular}{|c|c|c|c|c|c|c|}
\hline \multirow{2}{*}{ Comorbidities } & \multicolumn{3}{|c|}{ Univariate analysis } & \multicolumn{3}{|c|}{ Multivariate analysis } \\
\hline & OR & $95 \% \mathrm{Cl}$ & $\mathrm{p}$-value & $\mathrm{OR}$ & $95 \% \mathrm{Cl}$ & p-value \\
\hline Hypertension (yes vs no) & 2.210 & $1.156-4.224$ & 0.016 & 2.051 & $1.010-4.165$ & 0.047 \\
\hline Diabetes mellitus (yes vs no) & 2.223 & $1.130-4.374$ & 0.021 & 2.489 & $1.153-5.371$ & 0.020 \\
\hline Dyslipidemia (yes vs no) & 0.343 & $0.161-0.729$ & 0.005 & 0.291 & $0.128-0.663$ & 0.003 \\
\hline HBV infection (yes vs no) & 2.556 & $1.240-5.271$ & 0.011 & 2.447 & $1.124-5.325$ & 0.024 \\
\hline HCV infection (yes vs no) & 0.889 & $0.241-3.283$ & 0.860 & & & \\
\hline Alcoholism (yes vs no) & 2.062 & $0.335-12.703$ & 0.435 & & & \\
\hline
\end{tabular}

MAFLD, metabolic dysfunction-associated fatty liver disease; OR, odds ratio; $\mathrm{Cl}$, confidence interval; $\mathrm{HBV}$, hepatitis $\mathrm{B}$ virus; $\mathrm{HCV}$, hepatitis $\mathrm{C}$ virus.

disease. It is expected to include those with higher risk of disease progression that need specific treatment earlier for the metabolic dysfunction. However, the conceptional criteria for MAFLD have no histologic validation in real world so far, and there is only a study examining the clinical characteristics based on the ultrasonography and laboratory data. ${ }^{8}$ Liver biopsy, although not performed routinely, remains the gold standard for diagnosis, and is the only reliable method to determine the severity of inflammation and fibrosis accurately. ${ }^{10}$ This study is the first one to validate the new criteria for MAFLD based on both clinical and histologic information.

The definition of MAFLD is considered to include more patients because the exclusion of alcoholism and chronic liver disease is not required. In our study, the number of patients with MAFLD was greater than those with NAFLD, compatible with the aforementioned expectation. Those with alcoholism, HBV infection and HCV infection excluded by NAFLD criteria were included in the MAFLD group. The concept of concomitant MAFLD and other liver diseases (dual etiology fatty liver disease) was proposed because of the rising global prevalence of metabolic diseases, which may co-exist frequently with other etiologies contributing to hepatic steatosis. ${ }^{11}$ For example, the presence of HCV infection was reported to be associated with hepatic steatosis and metabolic syndrome. ${ }^{12}$ In the NAFLD criteria, the exclusion of these patients may lead to underdiagnosis and omit the timely management for the metabolic dysregulation. In our study, HBV infection, hypertension and DM were found to be independently associated with advanced fibrosis in our patients with MAFLD, compatible with the previous studies revealing that the presence of metabolic syndrome or diseases carried a high risk of hepatic fibrosis. ${ }^{13,14}$ Even in the non-obese populations, those with fatty liver disease had higher risks of DM and metabolic syndrome. ${ }^{15}$ Those with NAFLD-associated HCC were also known to be usually accompanied with metabolic disorders. ${ }^{16}$ However, the two comorbidities, HCV and alcoholism, were expected to be associated with advanced fibrosis but not in this study. The plausible expla- nation may be the small number of these subpopulations for statistical significance. Although the obeticholic acid is a potential candidate of pharmacotherapy for fatty liver disease in the future, ${ }^{17}$ lifestyle modifications to correct metabolic dysregulation remain the mainstream of treatment. ${ }^{18,19}$ The new criteria for MAFLD may help to select those with above treatable concurrent conditions.

There were 81 patients not included by the NAFLD criteria but meeting the MAFLD criteria in our study. We compared them with the other nine patients who met the NAFLD criteria but not the MAFLD criteria. The direct comparison helps clarify the major difference between the two distinct disease populations selected by different definitions. The patients in the MAFLD-only group had significantly lower platelet count and higher level of total bilirubin as compared with those in the NAFLD-only group, suggesting that patients with possibly higher degree of disease severity tend to be identified according to the novel MAFLD criteria for further management. In the comparison of histologic features, the NAS and the percentage of patients with advanced fibrosis were also significantly higher in the MAFLD-only group than that in the NAFLDonly group, confirming the nature of higher severity in patients with MAFLD rather than NAFLD by histology. Of note, the nine patients with NAFLD but not MAFLD had a mean NAS of 1.67 only, indicating less disease activity of fatty liver disease. 9 Those patients may be classified into socalled "alternative causes" of fatty liver disease in the future according to the consensus statement. ${ }^{6}$

This study was conducted in the HBV-endemic area and the prevalence reached $26.8 \%$ in our patients with MAFLD. Although there are several studies demonstrating the negative correlation between $\mathrm{HBV}$ infection and hepatic steatosis, ${ }^{20,21}$ those with HBV infection were excluded by the diagnostic criteria of NAFLD. In the analysis of factors associated with advanced fibrosis, the presence of HBV infection had a higher risk than the absence in our study. A previous study concluded that concurrent fatty liver proven by histology in HBV patients increases the risk of HCC. ${ }^{22}$ Another recent study using a large cohort of 
chronic hepatitis B patients found that those with concomitant NASH and chronic hepatitis B had more advanced fibrosis, mortalities and liver-related adverse outcomes. ${ }^{23}$ Those with HBV infection and concurrent metabolic factors were known to be associated with an increased risk of HCC than those without. ${ }^{24,25}$ Since the new criteria focuses on the metabolic dysfunction, it is expected to select more such patients in $\mathrm{HBV}$ population for early management to improve the long-term outcomes.

There were several strengths in our study. For the first time, we provide the histologic features and grading of MAFLD in the real world, with emphasis on the difference from NAFLD. In addition, the patients were collected in a recent decade (from 2009 to 2019), which means the baseline characteristics and prevalence of the metabolic diseases were closer to those in current population. Also, the HBV infection was included as a variable for analysis because it was conducted in the HBV-endemic area. There were also some limitations. First, this is a retrospective study conducted in a single tertiary center in Taiwan, and the results need to be validated in the Western countries. Second, the prevalence of MAFLD could not be evaluated in the general population because only patients with histology-proven hepatic steatosis were enrolled for analysis. Third, the number of patients with certain comorbidities, such as alcoholism and HCV, may be too small in our cohort to demonstrate the statistical significance in the correlation with advanced fibrosis. Studies in the future with larger number of biopsy-proven patients may help clarify this point.

In conclusion, the novel diagnostic criteria for MAFLD are useful and help identify patients with higher degree of disease severity and more treatable comorbidities for early intervention.

\section{CONFLICTS OF INTEREST}

No potential conflict of interest relevant to this article was reported.

\section{ACKNOWLEDGEMENTS}

This study was funded by the Ministry of Science and Technology and Ministry of Health and Welfare, Executive Yuan, Taiwan; National Taiwan University Hospital (NTUH 109-A149), Taipei, Taiwan.

\section{AUTHOR CONTRIBUTIONS}

Drafting of the article: S.C.H., C.J.L., H.J.S. Data acquisition: H.J.S., S.C.H. Interpretation of the data: C.J.L., S.C.H. Statistical analysis: S.C.H., H.J.S. Conception and design of study: C.J.L., S.C.H., H.J.S. Critical revision for important intellectual content and final approval of the manuscript: S.C.H., C.J.L., H.J.S., J.H.K., T.C.T., H.C.Y., T.H.S., P.J.C. Study supervision: C.J.L., J.H.K., P.J.C.

\section{ORCID}

Shang-Chin Huang

https://orcid.org/0000-0003-2507-9627

Hau-Jyun Su https://orcid.org/0000-0002-0244-9288

Jia-Horng Kao https://orcid.org/0000-0002-2442-7952

Tai-Chung Tseng https://orcid.org/0000-0003-0420-8311

Hung-Chih Yang https://orcid.org/0000-0003-3864-9895

Tung-Hung Su https://orcid.org/0000-0002-6747-7941

Pei-Jer Chen https://orcid.org/0000-0001-8316-3785

Chun-Jen Liu https://orcid.org/0000-0002-6202-0993

\section{REFERENCES}

1. Younossi ZM, Stepanova M, Afendy M, et al. Changes in the prevalence of the most common causes of chronic liver diseases in the United States from 1988 to 2008. Clin Gastroenterol Hepatol 2011;9:524-530.

2. Fan JG, Kim SU, Wong VW. New trends on obesity and NAFLD in Asia. J Hepatol 2017;67:862-873.

3. European Association for the Study of the Liver (EASL); European Association for the Study of Diabetes (EASD); European Association for the Study of Obesity (EASO). EASL-EASD-EASO Clinical Practice Guidelines for the management of non-alcoholic fatty liver disease. J Hepatol 2016;64:1388-1402.

4. Wong VW, Chan WK, Chitturi S, et al. Asia-Pacific Working Party on Non-alcoholic Fatty Liver Disease guidelines 2017Part 1: Definition, risk factors and assessment. J Gastroenterol Hepatol 2018;33:70-85.

5. Siddiqui MS, Harrison SA, Abdelmalek MF, et al. Case definitions for inclusion and analysis of endpoints in clinical trials for nonalcoholic steatohepatitis through the lens of regulatory science. Hepatology 2018;67:2001-2012.

6. Eslam M, Newsome PN, Sarin SK, et al. A new definition for metabolic dysfunction-associated fatty liver disease: an international expert consensus statement. J Hepatol 2020;73:202209.

7. Younossi ZM, Rinella ME, Sanyal A, et al. From NAFLD to 
MAFLD: implications of a premature change in terminology. Hepatology 2021;73:1194-1198.

8. Lin S, Huang J, Wang M, et al. Comparison of MAFLD and NAFLD diagnostic criteria in real world. Liver Int 2020;40:2082-2089.

9. Kleiner DE, Brunt EM, Van Natta M, et al. Design and validation of a histological scoring system for nonalcoholic fatty liver disease. Hepatology 2005;41:1313-1321.

10. Neuschwander-Tetri BA, Clark JM, Bass NM, et al. Clinical, laboratory and histological associations in adults with nonalcoholic fatty liver disease. Hepatology 2010;52:913-924.

11. Boyle M, Masson S, Anstee QM. The bidirectional impacts of alcohol consumption and the metabolic syndrome: cofactors for progressive fatty liver disease. J Hepatol 2018;68:251267.

12. Wang CC, Cheng PN, Kao JH. Systematic review: chronic viral hepatitis and metabolic derangement. Aliment Pharmacol Ther 2020;51:216-230

13. Marchesini G, Bugianesi E, Forlani G, et al. Nonalcoholic fatty liver, steatohepatitis, and the metabolic syndrome. Hepatology 2003;37:917-923.

14. Su HJ, Kao JH, Tseng TC, et al. Pathologic findings of patients with nonalcoholic fatty liver disease and the impact of concurrent hepatitis B virus infection in Taiwan. J Formos Med Assoc 2020;119:1476-1482.

15. Zou ZY, Wong VW, Fan JG. Epidemiology of nonalcoholic fatty liver disease in non-obese populations: meta-analytic assessment of its prevalence, genetic, metabolic, and histological profiles. J Dig Dis 2020;21:372-384.

16. Duan XY, Qiao L, Fan JG. Clinical features of nonalcoholic fatty liver disease-associated hepatocellular carcinoma. Hepatobiliary Pancreat Dis Int 2012;11:18-27.
17. Younossi ZM, Ratziu V, Loomba R, et al. Obeticholic acid for the treatment of non-alcoholic steatohepatitis: interim analysis from a multicentre, randomised, placebo-controlled phase 3 trial. Lancet 2019;394:2184-2196.

18. Wong VW, Wong GL, Chan RS, et al. Beneficial effects of lifestyle intervention in non-obese patients with non-alcoholic fatty liver disease. J Hepatol 2018;69:1349-1356.

19. Fan JG, Cao HX. Role of diet and nutritional management in non-alcoholic fatty liver disease. J Gastroenterol Hepatol 2013;28 Suppl 4:81-87.

20. Joo EJ, Chang Y, Yeom JS, Ryu S. Hepatitis B virus infection and decreased risk of nonalcoholic fatty liver disease: a cohort study. Hepatology 2017;65:828-835.

21. Zhu L, Jiang J, Zhai X, et al. Hepatitis B virus infection and risk of non-alcoholic fatty liver disease: a population-based cohort study. Liver Int 2019;39:70-80.

22. Chan AW, Wong GL, Chan HY, et al. Concurrent fatty liver increases risk of hepatocellular carcinoma among patients with chronic hepatitis B. J Gastroenterol Hepatol 2017;32:667-676.

23. Choi HS, Brouwer WP, Zanjir WM, et al. Nonalcoholic steatohepatitis is associated with liver-related outcomes and all-cause mortality in chronic hepatitis B. Hepatology 2020;71:539-548.

24. Yu MW, Lin CL, Liu CJ, Yang SH, Tseng YL, Wu CF. Influence of metabolic risk factors on risk of hepatocellular carcinoma and liver-related death in men with chronic hepatitis B: a large cohort study. Gastroenterology 2017;153:1006-1017.

25. Huang SF, Chang IC, Hong CC, et al. Metabolic risk factors are associated with non-hepatitis $\mathrm{B}$ non-hepatitis $\mathrm{C}$ hepatocellular carcinoma in Taiwan, an endemic area of chronic hepatitis B. Hepatol Commun 2018;2:747-759. 